\title{
Use of Twitter to monitor attitudes toward depression and schizophrenia: an exploratory study
}

Introduction: The paper reports on an exploratory study of the usefulness of Twitter for unobtrusive assessment of stigmatizing attitudes in the community. Materials and Methods: Tweets with the hashtags \#depression or \#schizophrenia posted on Twitter during a 7-day period were collected. Tweets were categorised based on their content and user information and also on the extent to which they indicated a stigmatising attitude towards depression or schizophrenia (stigmatising, personal experience of stigma, supportive, neutral, or antistigma). Tweets that indicated stigmatising attitudes or personal experiences of stigma were further grouped into the following subthemes: social distance, dangerousness, snap out of it, personal weakness, inaccurate beliefs, mocking or trivializing, and self-stigma. Results and Discussion: Tweets on depression mostly related to resources for consumers (34\%), or advertised services or products for individuals with depression (20\%). The majority of schizophrenia tweets aimed to increase awareness of schizophrenia $(29 \%)$ or reported on research findings (22\%). Tweets on depression were largely supportive $(65 \%)$ or neutral (27\%). A number of tweets were specifically anti-stigma (7\%). Less than $1 \%$ of tweets reflected stigmatising attitudes $(0.7 \%)$ or personal experience of stigma $(0.1 \%)$. More than one third of the tweets which reflected stigmatising attitudes were mocking or trivialising towards individuals with depression (37\%). The attitude that individuals with depression should "snap out of it" was evident in $30 \%$ of the stigmatising tweets. The majority of tweets relating to schizophrenia were categorised as supportive (42\%) or neutral (43\%). Almost $10 \%$ of tweets were explicitly anti-stigma. The percentage of tweets showing stigmatising attitudes was $5 \%$, while less than $1 \%$ of tweets described personal experiences of stigmatising attitudes towards individuals with schizophrenia. Of the tweets that indicated stigmatising 
attitudes, most reflected inaccurate beliefs about schizophrenia being multiple personality disorder $(52 \%)$ or mocked or trivialised individuals with schizophrenia (33\%). Conclusions: The study supports the use of analysis of Twitter content to unobtrusively measure attitudes towards mental illness, both supportive and stigmatising. The results of the study may be useful in assisting mental health promotion and advocacy organisations to provide information about resources and support, raise awareness and counter common stigmatising attitudes. 


\section{Use of Twitter to monitor attitudes toward depression and 2 schizophrenia: an exploratory study}

\section{Mental illness stigma and Twitter}

6 Nicola J Reavley§, Melbourne School of Population and Global Health, University of

7 Melbourne, Victoria, Australia

8 Pamela D Pilkington, School of Psychology, Faculty of Health Sciences, Australian

9 Catholic University, Victoria, Australia

$10{ }^{\text {s}}$ Corresponding author: Nicola J Reavley, Melbourne School of Population Health, 207

11 Bouverie St, Parkville, VIC 3010, Australia. Email: nnreavley@unimelb.edu.au, Ph:

$12+61390357628$. 


\section{Introduction}

14 Mental illness affects as many as one in five people in any 12-month period (Kessler

15 et al. 2007). Many people with a mental illness experience social and economic

16 hardship as a direct result of their illness. They must cope with their symptoms and

17 also with stigma and discrimination that result from misconceptions about these

18 illnesses (McNair et al. 2002; Corrigan et al. 2003). These issues are a key concern

19 as stigma may deter people with symptoms from seeking help due to

20 embarrassment or the belief that others will think badly of them (Gulliver, Griffiths

21 and Christensen 2012; Yap, Reavley and Jorm 2013). Stigma may also compound the

22 experience of psychological distress and can adversely affect personal relationships

23 and the ability to achieve educational and vocational goals (Wells et al. 1994; Link et

24 al. 1997; Corrigan 2004).

25 In an effort to better understand the factors predicting stigmatising attitudes to 26 mental illness, a number of research studies have focused on media coverage of

27 mental disorders (Morgan and Jorm 2009; McGinty et al. 2014). A study of

28 newspaper reporting on mental illness in the USA found that $39 \%$ of reports

29 concerned dangerousness and violence (Corrigan et al. 2005). There is evidence that

30 such reports can increase stigmatising attitudes, including beliefs about

31 dangerousness. Data from surveys conducted in Germany showed an increase in

32 beliefs about dangerousness after two widely-publicised attacks on politicians by

33 people with schizophrenia (Angermeyer and Matschinger 1996). In another German

34 study, reading a newspaper article about mental illness and violent crime increased

35 stigmatising attitudes (Dietrich et al. 2006). However, the majority of studies

36 assessing the role of media coverage of mental illness has focused on traditional

37 media, with relatively little attention paid to newer social media platforms such as

38 Facebook and Twitter (Egan and Moreno 2011; Moreno et al. 2011). 


\section{PeerJ Reviewing Manuscript}

39 Twitter is a popular social networking website that allows users to post brief statuses

40 up to 140 characters in length, referred to as tweets. Twitter enables social

41 communication and networking between users through retweeting (reposting other

42 users' tweets to one's own feed), responding to tweets, and following other users'

43 Twitter feeds. Hashtags are included in tweets to identify a theme and enable other

44 users to view related posts with the same hashtag. It is estimated that $18 \%$ of online

45 US adults use Twitter (Pew Internet \& American Life Project 2011), with similar

46 numbers reported in other developed countries (Office for National Statistics 2013).

47 Unless Twitter users mark their tweets as private, these are public, making Twitter a

48 potentially valuable source of information about the views of people on a range of

49 topics. A number of studies have analysed Twitter content on health-related topics,

50 including an influenza outbreak (Chew and Eysenbach 2010), problem drinking

51 (West et al. 2012), dental pain (Heaivilin et al. 2011), palliative medicine (Nwosu et

52 al. 2014), physical activity (Zhang et al. 2013), dementia (Robillard et al. 2013),

53 vaccination (Love et al. 2013), and breast cancer (Thackeray et al. 2013). Martinez-

54 Perez et al. (2014) analysed the purposes and functions of Facebook and Twitter

55 groups for different mental illnesses, including depression. The results showed that

56 individuals with mental illness utilise online social networks to obtain information,

57 access support, and raise awareness. However, to our knowledge, the current study

58 is the first study to conduct a detailed analysis of Twitter content as it relates to

59 attitudes to mental illness. The aim of the study is to provide insights into how

60 Twitter users share information about depression and schizophrenia, the type of

61 information shared, and the relative proportions of supportive or stigmatising

62 attitudes in that content. The study offers the opportunity for unobtrusive attitude

63 measurement (Lee 2000), something which is currently lacking in much

64 conventional stigma research, which uses self-report attitude scales that run the risk 


\section{PeerJ Reviewing Manuscript}

65 of social desirability bias because the responses are given to a mental health

66 researcher.

\section{Method}

68 A qualitative analysis was conducted to explore supportive or $\mathrm{f}$ stigmatising

69 attitudes towards depression and schizophrenia on Twitter.

70 Data extraction

71 The second author retrieved all available English-language tweets that included the

72 hashtags \#depression or \#schizophrenia posted on Twitter (http://www.twitter.com)

73 twice daily for a 7-day period between the $27^{\text {th }}$ of November and the $4^{\text {th }}$ of December

74 2013. Twitter's advanced search function was used to limit the tweets to those in

75 English. The data were retrieved using NCapture for Chrome (QSR International

76 2012), a web browser extension that captures web pages, online PDFs, and social

77 media for analysis in NVivo 10 (QSR International 2012).

78 Accessing tweets directly through http://www.twitter.com provides access to what is

79 referred to as the Streaming Application Programing Interface (API). Twitter reports

80 that only $1 \%$ of existing tweets are available through the Streaming API. Access to

81 all tweets is gained through what is termed the "firehose", which is costly to access,

82 and requires sufficient servers, network availability, and disc space. Morstatter et al.

$83(2013)$ found that more than $40 \%$ of tweets in the "firehose" were available to the

84 Streaming API when data were accessed on a daily basis. Therefore, we captured

85 tweets twice daily to maximise our access to the available tweets.

86 The data retrieved included the tweet and related information (time and date

87 tweeted, number of retweets, hashtags, and mentions), as well as information about

88 the user (username, brief bio, location), and the extent of their Twitter use (number

89 of followers, number following, and total number of tweets). We visually inspected 
90 the time and date of each tweet to confirm that all tweets available during the time

91 period were captured continuously. Tweets were included in the analyses if they

92 referred to depression or schizophrenia as mental illnesses. Tweets were excluded

93 based on the following criteria: a) focused on a topic other than depression or

94 schizophrenia; b) referred to temporary levels of depressive affect; c) referred to

95 economic, historical, or meteorological depression (e.g., tropical depression, which is

96 a type of cyclone); d) could not be accurately coded due to lack of context or the

97 use of hashtags only; or e) was in a language other than English. Ethics approval

98 was not obtained as this is not needed for content analysis of publicly available

99 information.

\section{Content analysis}

101 A content analysis of the tweets was conducted using Nvivo 10. The second author

102 coded the tweets based on the extent to which they indicated a particular attitude

103 towards depression or schizophrenia (stigmatising, personal experience of stigma,

104 supportive, neutral, or anti-stigma). Tweets that indicated stigmatising attitudes or

105 personal experiences of stigma were further grouped into the following subthemes:

106 social distance, dangerousness, snap out of it, personal weakness, inaccurate

107 beliefs, mocking or trivializing, and self-stigma. These categories were developed by

108 consensus and are based on previous research investigating stigmatizing attitudes,

109 which has shown that stigmatising attitudes can be grouped into categories,

110 including desire for social distance from the person, beliefs that mental illness is due

111 to personal weakness and that people with mental illness are dangerous (Link 1987;

112 Griffiths, Christensen and Jorm 2008; Reavley and Jorm 2012).

113 Tweets were also categorised based on their content and user information. The

114 following content themes emerged: 1) personal experience of mental illness, 2) 
115 awareness promotion, 3) research findings, 4) resources for consumers 5)

116 advertising, 6) news media, 7) personal opinion or dyadic interaction.

117 Users were coded for type (individuals, consumer, health professional, organisation,

118 or advocate) and location based on their username and biographic information.

119 Table 1 provides theme definitions and provides examples of tweets that are

120 representative of the tweets coded within each theme. Both authors coded 5 to $10 \%$

121 of the tweets and users to ensure accurate coding. Initial levels of agreement

122 ranged between 67 to $80 \%$. Discrepancies were resolved through discussion and

123 consultation with the third person.

\section{Results}

125 A total of 7295 depression tweets and 500 schizophrenia tweets were retrieved from

126 Twitter over the 7-day period. After applying the inclusion and exclusion criteria,

1275907 depression tweets (81\%) and 451 schizophrenia tweets (90\%) were included in

128 the analyses. NCapture identified between 24 and $29 \%$ of the included tweets as

129 retweets. The vast majority of tweets included links to websites (75\% to $78 \%$ ).

\section{Users}

131 Over the 7-day period, 2019 users tweeted using the depression hashtag, while 230

132 users tweeted using the schizophrenia hashtag.

\section{Location}

134 Location data was available for $63 \%$ of users. The majority of users who listed their

135 location were from the United States of America (31\%), followed by the United

136 Kingdom (15\%), Canada (8\%), and Australia (3\%). The remaining $6 \%$ of tweeters

137 were located in Europe, Asia, the Middle East, Africa, South America, and New

138 Zealand. 


\section{PeerJ Reviewing Manuscript}

139 User type

140 Users tweeting about depression were mostly consumers (35\%), followed by

141 individuals (unspecified) (27\%), organisations (25\%), health professionals (9\%), and

142 mental health advocates (3\%). In contrast, the majority of users tweeting about

143 schizophrenia were individuals (54\%), followed by organisations ( $25 \%)$, health

144 professionals (8\%), mental health advocates (8\%), and consumers (4\%).

\section{Content}

146 The content of the tweets by mental illness is presented in Figure 1.

147 Depression: Tweets on depression mostly related to resources for consumers (34\%;

1482011 tweets) or advertised services or products for individuals with depression

149 (20\%; 1201 tweets). The remaining tweets described personal experiences with

150 depression (16\%; 977 tweets), promoted awareness of depression (15\%; 917

151 tweets), linked to news media articles on depression (6\%; 354 tweets), reported

152 research findings (5\%; 311 tweets), or described an individual's personal opinion or

153 view (2\%; 136 tweets). The hashtags which most commonly accompanied the

154 hashtag \#depression were \#anxiety, \#mentalhealth, \#bipolar, \#stress, and

155 \#suicide.

156 Schizophrenia: The majority of schizophrenia tweets aimed to increase awareness of

157 schizophrenia (29\%; 132 tweets) or reported on research findings (22\%; 100

158 tweets). Tweets providing resources, advice, or support for people with

159 schizophrenia accounted for $17 \%$ of the tweets ( 76 tweets). Twelve percent of

160 tweets described personal opinions (54 tweets), while 10\% described personal

161 experiences with schizophrenia (47 tweets). A small number of tweets consisted of

162 advertising (7\%; 34 tweets) and news media articles (2\% 8 tweets). The hashtags

163 which most commonly accompanied the hashtag \#schizophrenia were \#mental, 


\section{PeerJ Reviewing Manuscript}

164 \#health, \#mentalhealth, \#bcss, \#psych. The hashtag \#bcss refers to the British

165 Columbia Schizophrenia Society.

\section{Attitudes towards depression or schizophrenia}

167 The numbers of stigmatising attitudes by mental illness is shown in Figure 2. Figure

1683 presents the types of stigmatising attitudes that were evident.

169 Depression: Tweets on depression were largely supportive (65\%; 3839 tweets) or

170 neutral (27\%; 1600 tweets). A number of tweets were specifically anti-stigma (7\%;

171421 tweets). Less than $1 \%$ of tweets reflected stigmatising attitudes $(0.7 \% ; 43$

172 tweets) or personal experience of stigma ( $<0.1 \% ; 4$ tweets). More than one third of

173 the tweets which reflected stigmatising attitudes were mocking or trivialising

174 towards individuals with depression (37\%; 16 tweets). The attitude that individuals

175 with depression should "snap out of it" was evident in 30\% (13 tweets) of the

176 stigmatising tweets. The belief that people with depression are weak was displayed

177 in $21 \%$ (9 tweets) of tweets. A further $12 \%$ (5 tweets) of the tweets suggested self-

178 stigma.

179 Schizophrenia: The majority of tweets relating to schizophrenia were categorised as

180 neutral (43\%; 193 tweets) or supportive (42\%; 191 tweets). Almost 10\% of tweets

181 were explicitly anti-stigma (44 tweets). The percentage of stigmatising attitudes was

$1825 \%$ (21 tweets), while less than $1 \%$ of tweets described personal experiences of

183 stigmatising attitudes towards individuals with schizophrenia ( 2 tweets). Of the

184 tweets that indicated stigmatising attitudes, most reflected inaccurate beliefs about

185 schizophrenia being multiple personality disorder (52\%; 11 tweets) or mocked or

186 trivialised individuals with schizophrenia (33\%; 7 tweets). The remaining tweets

187 indicated an unwillingness to be in social contact with people with schizophrenia 


\section{PeerJ Reviewing Manuscript}

188 (5\%; 1 tweet), a belief that individuals with schizophrenia are dangerous (5\%; 1

189 tweet), or were suggestive of self-stigma (5\%; 1 tweet).

190 Discussion

191 Content analysis of Twitter information about depression and schizophrenia can

192 provide insights into the type of information shared, who is sharing the information,

193 and the types of attitudes displayed, whether supportive or stigmatising. An analysis

194 of tweets with the hashtags \#depression or \#schizophrenia posted on Twitter during

195 a 7-day period revealed that most tweets for both illnesses were supportive or

196 neutral, although stigmatising attitudes were more prevalent in the schizophrenia

197 tweets. More than one third of the depression tweets which reflected stigmatising

198 attitudes were mocking or trivialising towards individuals with depression and the

199 belief that individuals with depression should "snap out of it" was evident in almost

200 one third. To some extent, these findings reflect surveys assessing stigmatising

201 attitudes towards people with depression, which have shown the beliefs that people

202 are weak rather than sick are relatively common (Reavley and Jorm 2011), although

203 views about the dangerousness of people with depression, which are relatively

204 common in surveys was not reflected in the tweets. Of the schizophrenia tweets that

205 indicated stigmatising attitudes, just over half reflected inaccurate beliefs about

206 schizophrenia being multiple personality disorder while one third mocked or

207 trivialised individuals with schizophrenia.

208 In terms of the type of information, the results of the current study showed that

209 tweets on depression mostly related to resources for consumers or advertised

210 services or products for individuals with depression, whereas the majority of

211 schizophrenia tweets aimed to increase awareness of schizophrenia or reported on

212 research findings. These differences are likely to relate to the higher prevalence of

213 depression than schizophrenia and therefore the potentially greater market for 
214 products and services. These findings may be compared to those of a recent study

215 by Martinez-Perez et al. (2014) who analysed the purposes and functions of

216 Facebook and Twitter groups for different mental illnesses, including depression.

217 They classified groups according to whether they were support groups, self-help

218 groups, advocacy and awareness groups and fundraising groups. They concluded

219 that self-help groups were the most common category (64\%), followed by support

220 groups (15\%), and advocacy and awareness groups (10\%). They also identified the

221 creators of the groups, listing the following categories: associations, societies,

222 foundations, collectives, individuals, companies, caregivers, and specialists. Over

$22370 \%$ of creators were identified as individuals. However, they did not do further

224 analysis on the content of the tweets.

225 As far as we are aware, this is the first study to assess the content of tweets on the

226 topic of mental illness. Strengths of the study include the opportunity for

227 unobtrusive measurement of freely volunteered views (Lee 2000). Results of such

228 studies are less likely to be affected by social desirability bias than responses to

229 surveys, which are more commonly used to assess stigmatising attitudes

230 (Angermeyer and Matschinger 2004; Reavley and Jorm 2011).

231 With the increasing prominence of social media, the results of the study may be

232 useful in assisting mental health promotion and advocacy organisations to provide

233 information about resources and support, raise awareness and reduce stigma.

234 Twitter may also be an increasingly useful resource for people seeking health

235 information on the internet as a number of tweets provided links to health

236 information. There is evidence that the quality of online information is improving,

237 particularly that relating to affective disorders, and it is likely that online information

238 and interventions will continue to become more important (Reavley and Jorm 2010).

239 In addition, advocacy organisations may want to specifically counter common 


\section{PeerJ Reviewing Manuscript}

240 stigmatising attitudes such as beliefs about weakness in people with depression or

241 beliefs about 'split personality' in people with schizophrenia. A recent campaign to

242 raise awareness and counter the use of stigmatising terms for people with

243 intellectual disabilities has taken the approach of directly responding to celebrities

244 who use these terms (Sibley 2014)

245 The main limitation of the study is our inability to determine what proportion of

246 existing tweets referring to depression and anxiety were analysed. Comparisons

247 between the Streaming API and the "firehose" suggest that the Streaming API may

248 not sufficiently represent the activity on Twitter as a whole (Morstatter, Pfeffer et al.

249 2013). Despite this, we believe that it was appropriate to access the Streaming API

250 as the significant costs involved in accessing the "firehose" would not yield

251 proportionate benefits. We analysed a sufficient number of tweets to reach data

252 saturation. Furthermore, the Streaming API is available to the public, and therefore

253 representative of how the average person experiences Twitter.

254 A second potential limitation is our focus on tweets using the depression and

255 schizophrenia hashtags. This may have meant that content related to other

256 hashtags or slang terms such as "schizo" (which people with stigmatising attitudes

257 are potentially more likely to use) may have been missed. Moreover, the anonymity

258 of most users makes it impossible to verify the authenticity of content or to assess

259 stigmatising attitudes according to age, gender, or location. Finally, these findings

260 cannot be generalised to communities outside Twitter.

261 Given the speed of reactions to events on social media, it would be useful for future

262 research to compare attitudes before and after significant events, for which it is not

263 possible to anticipate a 'pre-test' (e.g., a mass shooting that involves a person with 
264 a mental illness) as this may also provide useful information on stigmatising

265 attitudes.

\section{Conclusion}

267 The study supports the use of analysis of Twitter content to unobtrusively measure 268 attitudes towards mental illness, both supportive and stigmatising. The results of the

269 study may be useful in assisting mental health promotion and advocacy

270 organisations to provide information about resources and support, raise awareness

271 and counter common stigmatising attitudes.

\section{References}

273 Angermeyer, M. C. and H. Matschinger (1996). "The effect of violent attacks by schizophrenic

274 persons on the attitude of the public towards the mentally ill." Social Science and

$275 \quad$ Medicine 43: 1721-1728.

276 Angermeyer, M. C. and H. Matschinger (2004). "Public attitudes to people with depression: have 277 there been any changes over the last decade?" Journal of Affective Disorders 83: 177-182.

278 Chew, C. and G. Eysenbach (2010). "Pandemics in the age of Twitter: content analysis of Tweets 279 during the 2009 H1N1 outbreak." PLoS ONE 5: e14118.

280 Corrigan, P. (2004). "How stigma interferes with mental health care." American Psychologist 59:

$281 \quad 614-625$.

282 Corrigan, P., V. Thompson, D. Lambert, Y. Sangster, J. G. Noel and J. Campbell (2003).

283 "Perceptions of discrimination among persons with serious mental illness." Psychiatric 284 Services 54: 1105-1110.

285 Corrigan, P. W., A. C. Watson, G. Gracia, N. Slopen, K. Rasinski and L. L. Hall (2005).

$286 \quad$ "Newspaper stories as measures of structural stigma." Psychiatric Services 56: 551-556. 
287 Dietrich, S., D. Heider, H. Matschinger and M. C. Angermeyer (2006). "Influence of newspaper 288 reporting on adolescents' attitudes toward people with mental illness." Social Psychiatry 289 and Psychiatric Epidemiology 41: 318-322.

290 Egan, K. G. and M. A. Moreno (2011). "Prevalence of stress references on college freshmen 291 Facebook profiles." Computers, Informatics, Nursing 29: 586-592.

292 Griffiths, K. M., H. Christensen and A. F. Jorm (2008). "Predictors of depression stigma." BMC $293 \quad$ Psychiatry 8: 25.

294 Gulliver, A., K. M. Griffiths and H. Christensen (2012). "Barriers and facilitators to mental health 295 help-seeking for young elite athletes: a qualitative study." BMC Psychiatry 12: 157.

296 Heaivilin, N., B. Gerbert, J. E. Page and J. L. Gibbs (2011). "Public health surveillance of dental 297 pain via Twitter." Journal of Dental Research 90: 1047-1051.

298 Kessler, R. C., M. Angermeyer, J. C. Anthony, R. De Graaf, K. Demyttenaere, I. Gasquet, G. De 299 Girolamo, S. Gluzman, O. Gureje, J. M. Haro, N. Kawakami, A. Karam, D. Levinson, M. 300 E. Medina Mora, M. A. Oakley Browne, J. Posada-Villa, D. J. Stein, C. H. Adley Tsang, 301 S. Aguilar-Gaxiola, J. Alonso, S. Lee, S. Heeringa, B. E. Pennell, P. Berglund, M. J. Gruber, M. Petukhova, S. Chatterji and T. B. Ustun (2007). "Lifetime prevalence and age-

305 Lee, R. M. (2000). Unobtrusive Methods in Social Research. Buckingham, UK, Open University $306 \quad$ Press.

307 Link, B. G. (1987). "Understanding labeling effects in the area of mental disorders: An 308 assessment of the effects of expectations of rejection." American Sociological Review 52: 309 96-112. 
310 Link, B. G., E. L. Struening, M. Rahav, J. C. Phelan and L. Nuttbrock (1997). "On stigma and its

311 consequences: evidence from a longitudinal study of men with dual diagnoses of mental

312 illness and substance abuse." Journal of Health and Social Behavior 38: 177-190.

313 Love, B., I. Himelboim, A. Holton and K. Stewart (2013). "Twitter as a source of vaccination 314 information: content drivers and what they are saying." American Journal of Infection

$315 \quad$ Control 41: 568-570.

316 Martinez-Perez, B., I. de la Torre-Diez, B. Bargiela-Florez, M. Lopez-Coronado and J. J.

317 Rodrigues (2014). "Content analysis of neurodegenerative and mental diseases social 318 groups." Health Informatics Journal.

319 McGinty, E. E., D. W. Webster, M. Jarlenski and C. L. Barry (2014). "News media framing of 320 serious mental illness and gun violence in the United States, 1997-2012." American $321 \quad$ Journal of Public Health 104: 406-413.

322 McNair, B. G., N. J. Highet, I. B. Hickie and T. A. Davenport (2002). "Exploring the perspectives 323 of people whose lives have been affected by depression." Medical Journal of Australia 324176 Suppl: S69-76.

325 Moreno, M. A., L. A. Jelenchick, K. G. Egan, E. Cox, H. Young, K. E. Gannon and T. Becker 326 (2011). "Feeling bad on Facebook: depression disclosures by college students on a social 327 networking site." Depression and Anxiety 28: 447-455.

328 Morgan, A. J. and A. F. Jorm (2009). "Recall of news stories about mental illness by Australian 329 youth: associations with help-seeking attitudes and stigma." $330 \quad$ Journal of Psychiatry 43: 866-872.

331 Morstatter, F., J. Pfeffer, H. Liu and K. M. Carley (2013). Is the sample good enough? Comparing 332 data from Twitter's Streaming API with twitter's firehose. Seventh International AAAI 333 Conference on Weblogs and Social Media, Massachusetts, USA. 
334 Nwosu, A., M. Debattista, C. Rooney and S. Mason (2014). "Social media and palliative

335

336 medicine: a two year analysis of global twitter data." BMJ Support Palliat Care 4 Suppl 1: A75.

Office for National Statistics (2013). Social Networking: The UK as a Leader in Europe. London, 338 UK, Office for National Statistics,.

339 Pew Internet \& American Life Project (2011). Health Topics. Washington DC, Pew Internet.

340 QSR International (2012). NCapture (Version 1.0.136.0). Doncaster, Australia, QSR International $341 \quad$ Pty Ltd.

342 QSR International (2012). NVivo qualitative data analysis software. Doncaster, Australia, QSR 343 International Pty Ltd.

344 Reavley, N. J. and A. F. Jorm (2010). "The quality of mental disorder information websites: A 345 review." Patient Education and Counseling 85: e16-25.

346 Reavley, N. J. and A. F. Jorm (2011). "Stigmatizing attitudes towards people with mental 347 disorders: Findings from an Australian National Survey of Mental Health Literacy and 348 Stigma." Australian and New Zealand Journal of Psychiatry 48: 1086-1093.

349 Reavley, N. J. and A. F. Jorm (2012). "Stigmatizing attitudes towards people with mental 350 disorders: changes in Australia over 8 years." Psychiatry Research 197: 302-306.

351 Robillard, J. M., T. W. Johnson, C. Hennessey, B. L. Beattie and J. Illes (2013). "Aging 2.0:

352 health information about dementia on Twitter." PLoS ONE 8: e69861.

353 Sibley, J. (2014). "TweetRethink case study." from http://www.youtube.com/watch? $354 \quad \mathrm{v}=$ or76 $\mathrm{xmHfN} 3$ Y \&feature $=$ youtu.be.

355 Thackeray, R., S. H. Burton, C. Giraud-Carrier, S. Rollins and C. R. Draper (2013). "Using 356 Twitter for breast cancer prevention: an analysis of breast cancer awareness month." $\underline{B M C}$ $357 \quad$ Cancer 13: 508. 
358 Wells, J. E., L. N. Robins, J. A. Bushnell, D. Jarosz and M. A. Oakley-Browne (1994). "Perceived

359 barriers to care in St. Louis (USA) and Christchurch (NZ): reasons for not seeking

360 professional help for psychological distress." Social Psychiatry and Psychiatric

$361 \quad$ Epidemiology 29: 155-164.

362 West, J. H., P. C. Hall, K. Prier, C. L. Hanson, C. Giraud-Carrier, E. S. Neeley and M. D. Barnes

363 (2012). "Temporal variability of problem drinking on Twitter." Open J Prev Med 2: 43-48.

364 Yap, M. B., N. Reavley and A. F. Jorm (2013). "Where would young people seek help for mental

365 disorders and what stops them? Findings from an Australian national survey." Journal of

366 Affective Disorders 147: 255-261.

367 Zhang, N., S. Campo, K. F. Janz, P. Eckler, J. Yang, L. G. Snetselaar and A. Signorini (2013).

368 "Electronic word of mouth on twitter about physical activity in the United States:

369 exploratory infodemiology study." Journal of Medical Internet Research 15: e261. 


\section{Figure 1}

Figure 1 Content of tweets by mental illness

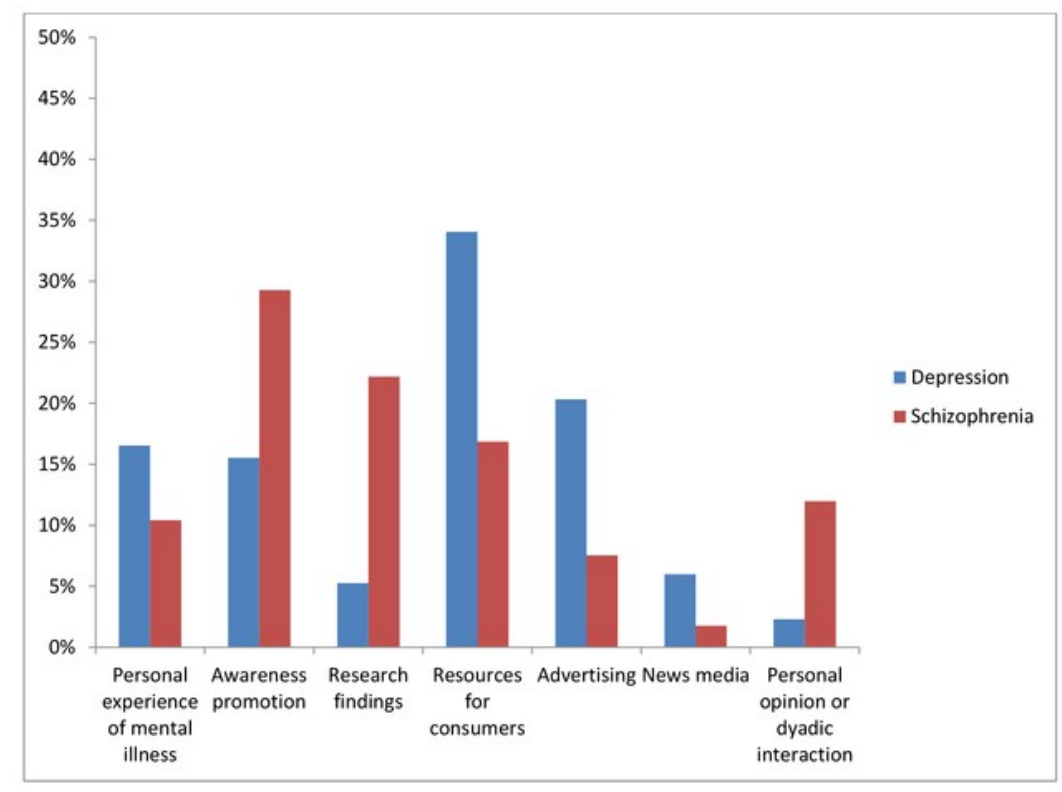




\section{Figure 2}

Figure 2 Types of attitudes by mental illness

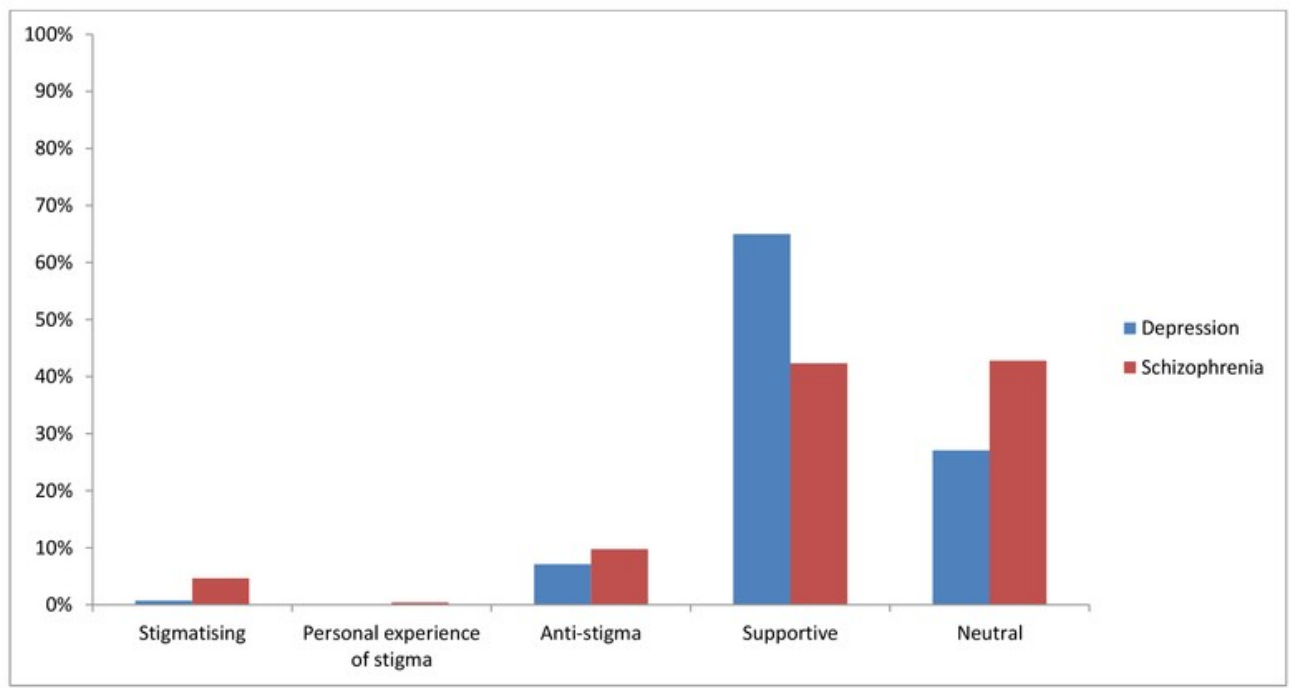




\section{Table 1 (on next page)}

Table 1 Theme definitions and representative tweets 


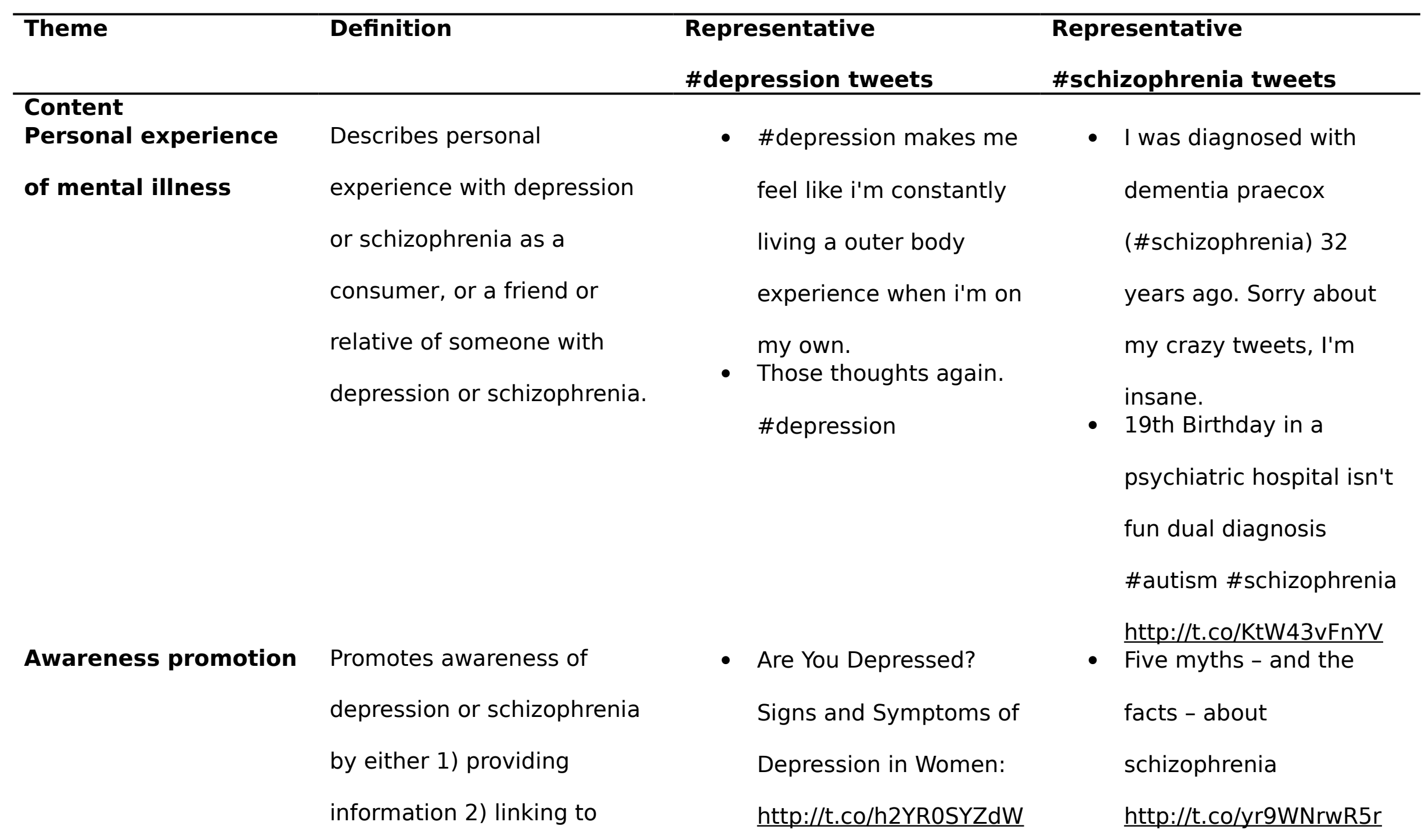


information on a website

(e.g., blog, news article, e-

book, or YouTube video) or 3)

depression or schizophrenia.

\section{Resources for}

consumers

Provides resources, advice, or

support specifically for encouraging discussion about

\#depression Please RT

- What does \#depression

feel like? Mental Health

\section{Advocate}

@ArthurGallant27 offers

his perspective here:

http://t.co/CTIUFI6dez

consumers or friends and

relatives of someone with

depression or schizophrenia;

or encourages social

networking between

consumers.
- RT @Daniel_L_Baker: If you're suffering from \#depression or know someone who is,

download my FREE e-

book at

http://t.co/oi3n2PobPL

- How to Exercise When
\#Stigma \#Schizophrenia

\#MentalHealth \#1/n4

- Check out "Schizophrenia

Documentary" on

\#Vimeo

http://t.co/8Zq0TV5ih]

\#mentalillness

\#schizophrenia

\#depression

\#breakdown

\#MentalHealth

- 6 tips for families dealing

with \#schizophrenia

http://t.co/pIBFQGYTCs

- Information for young people recovering from psychosis - This booklet was designed for...

http://t.co/ziN5gAtNxr 


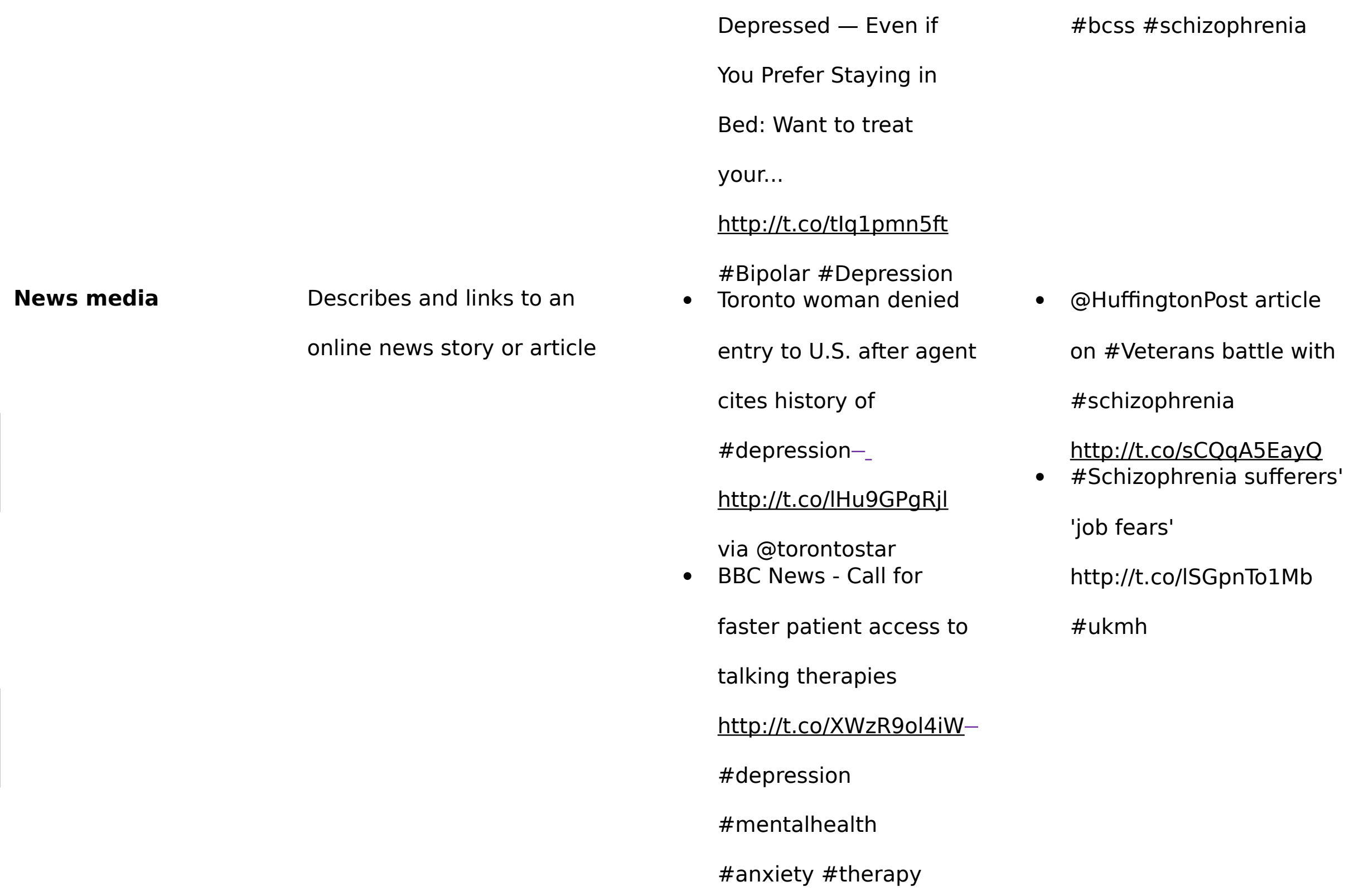




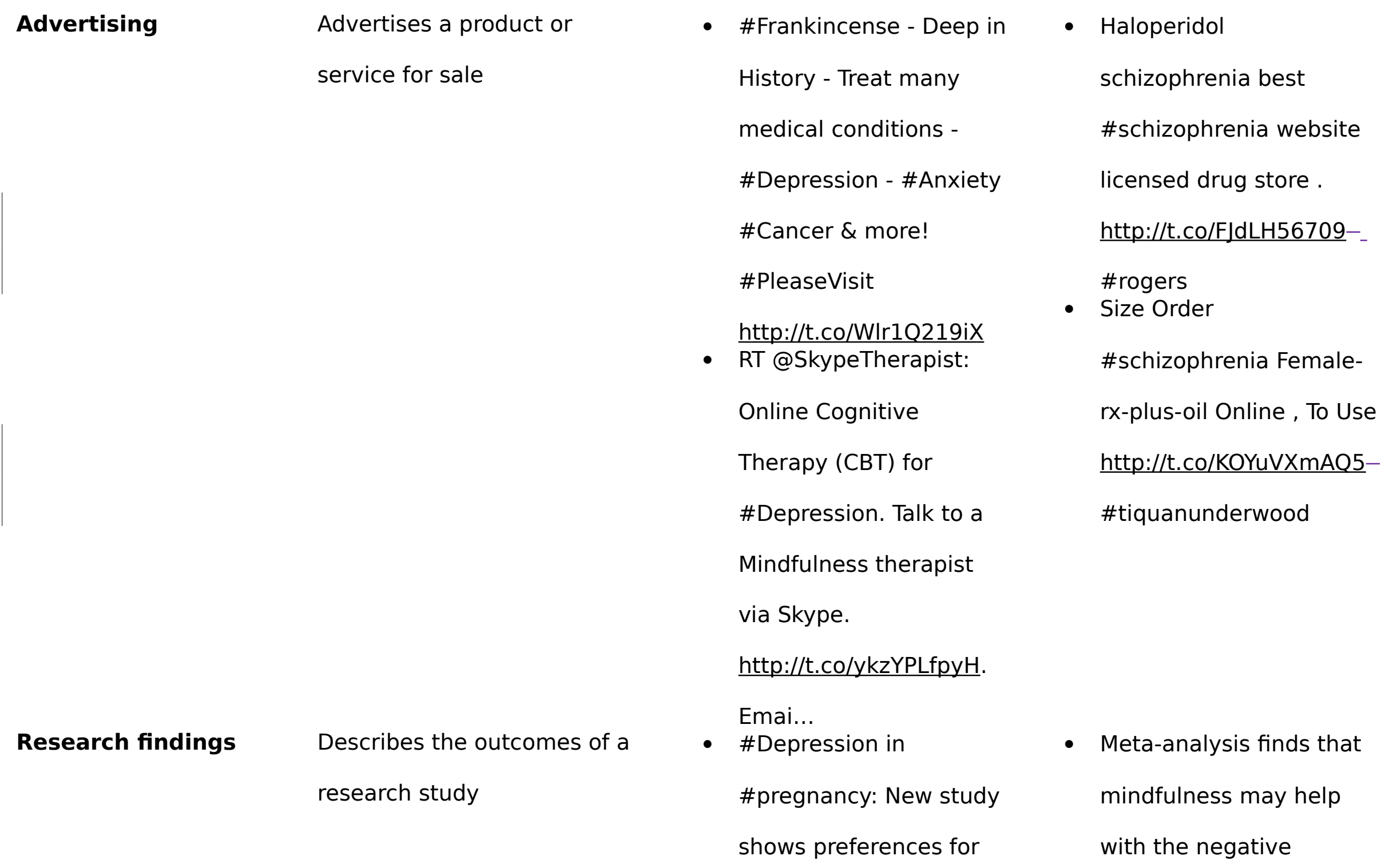




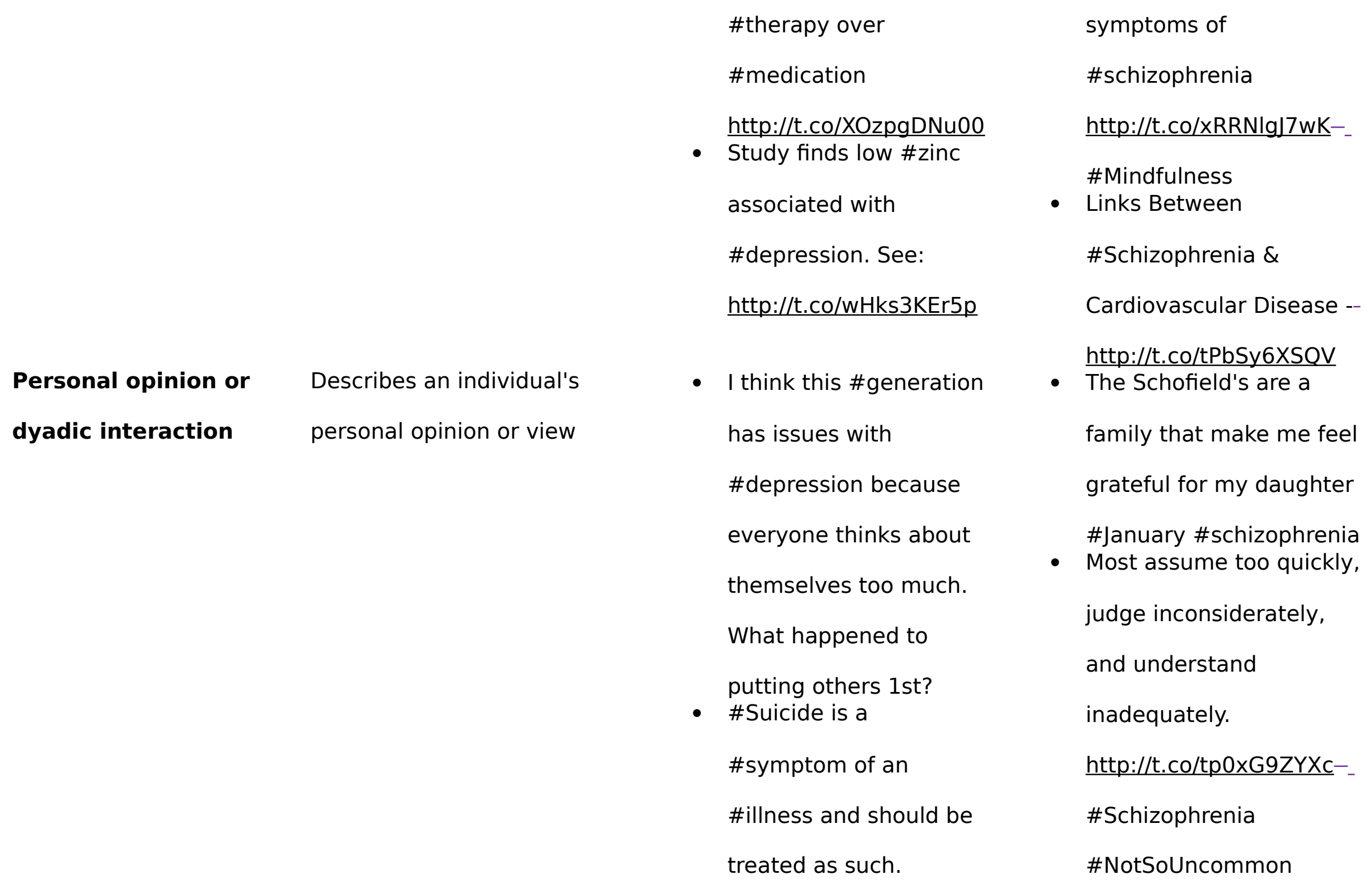


\#depression

\section{Attitude \\ Stigmatising}

Personal experience of stigma
Indicates a negative attitude

towards people with

depression/schizophrenia

Describes a personal

experience of stigmatising

attitudes or discrimination
- Wanna know the secret

of \#depression? People

only have it if they

know they've become a

f***ing \#loser, the rest

is just an \#excuse \#stfu

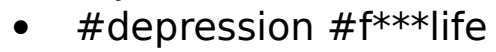

\#crazy \#killyourself

\#crazy \#whore \#retard

\#idiot \#psycho

\#horrible \#dumb

\#stupid \#bitch \#emo

http://t.co/aUr63r5ax8

- My husband suffered

from \#depression \&

\#anxiety. I was amazed
- Schizophrenia:

Medicine's mystery -

Society's shame

\#schizophrenia

http://t.co/BGIVJuSe2/

- Rihanna gets along with

the voices in her head

\#schizophrenia

\#helprequired

- @seniorchuffy @dROP2RIPPLE

@andrewnorthcbc people 
towards

depression/schizophrenia on how many said he

should "just get over it."

It's a disease, not a

choice.

- \#quote \#patience

\#depression

pic,twitter,comtRB98Lb

ake, People make

assumptions about us

and that really gets to

me,"

Explicitly promotes the

reduction of stigma towards

people with depression/

schizophrenia
- RT @Daniel_L_Baker:

People can't just “snap

out of \#depression". It

doesn't work like that. If

it did, don't you think

they would?

- Are you sick of being with SMI left out shows

that there's little

engagement w/people

with \#Schizophrenia

here

- \#MentalHealth

\#Schizophrenia Many people are not on my

side most take

advantage of me and

many are still out to...

http://t.co/3yS8IvS61U

- And if you think that

people with

\#schizophrenia (like me)

or other mental health

conditions are "psycho"

or dangerous, shame on 


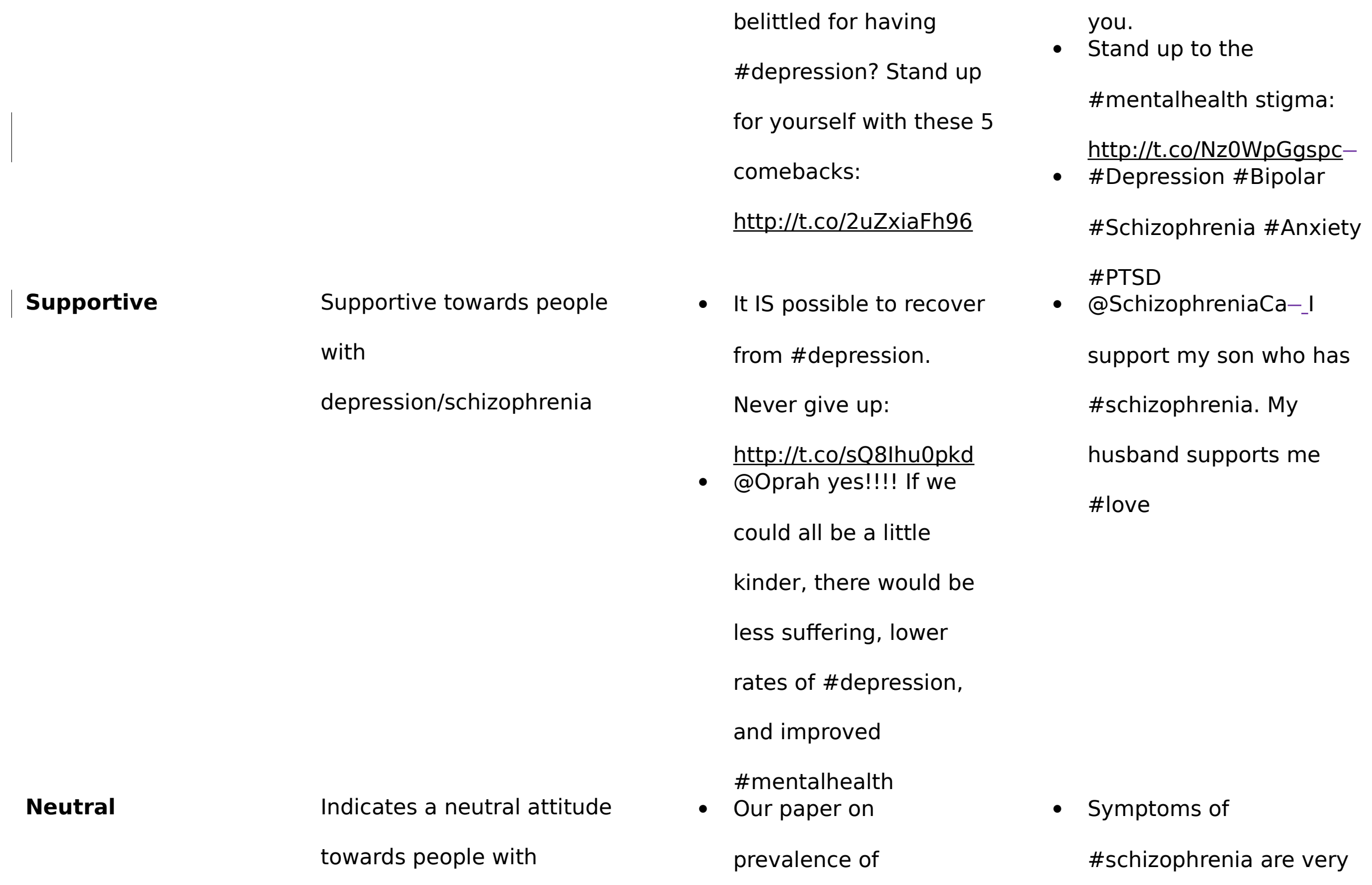


depression/ schizophrenia

\section{Type of Stigma}

Social distance \#depression and

\#BipolarDisorder within

UK Biobank, just

published @PLOSONE

http://t.co/6nFj...

- Sometimes you just hit

a point where life just

feels like it's too hard

and you just want to

give up. \#depression

\#fml

Unwillingness to have social

contact with people with

depression/ schizophrenia common, raising

questions about how

distinct the diagnosis is.

http://t.co/6yZSOto8dK

\#m..

- Just submitted my third

essay!! \#relax

\#psychology

\#schizophrenia

- The Schofield's are a

family that make me feel

grateful for my daughter

\#January \#schizophrenia

- Seeing these poor babies

with these illnesses

makes me want to hold 


\section{Dangerousness}

Snap out of it

Personal weakness
Belief that people with

depression/schizophrenia are

dangerous

Belief that people with

depression/schizophrenia

could snap out of it

Belief that

depression/schizophrenia is a my boys and thank god

that they are

healthy\#elhamdallah

\#schizophrenia

- Untreated

\#Schizophrenia Boosts

Likelihood of Future

Violence Among

Prisoners

http://t.co/vWImtU6CQd

- How low can a person

go b4 I can scold the

person to wake up get a

job get a life?

\#depression \#stubborn

\#pride preventing

accepting help

- \#depression is a crisis

of confidence 
sign of personal weakness

Inaccurate beliefs Indicates a lack of knowledge

or inaccurate understanding

of depression/schizophrenia

is. E.g., That schizophrenia

refers to having multiple

personalities.

Mocking or trivialising Rude, derogotary, or

trivialising towards people

with depression

/schizophrenia

Self-stigma

Indicates that the consumer
http://t.co/q3jumRiTZ9

- Btw, Katie is my other

personality

\#schizophrenia

- Getting ready to hang

out with myself!

\#schizophrenia

- Tyson: To get rid of

depression you sit in a

cupboard and pee your

pants!

Creager: Noooooooo!

\#depression

has internalised a

stigmatising attitude towards

people with
- Wish it wasn't so hard

to ask for help

\#depression

\#wordsiwillneversay 
PeerJ Reviewing Manuscript

depression/schizophrenia

\#Truth

*No tweets identified for this theme 


\section{Figure 3}

Figure 3 Types of stigmatising attitudes by mental illness

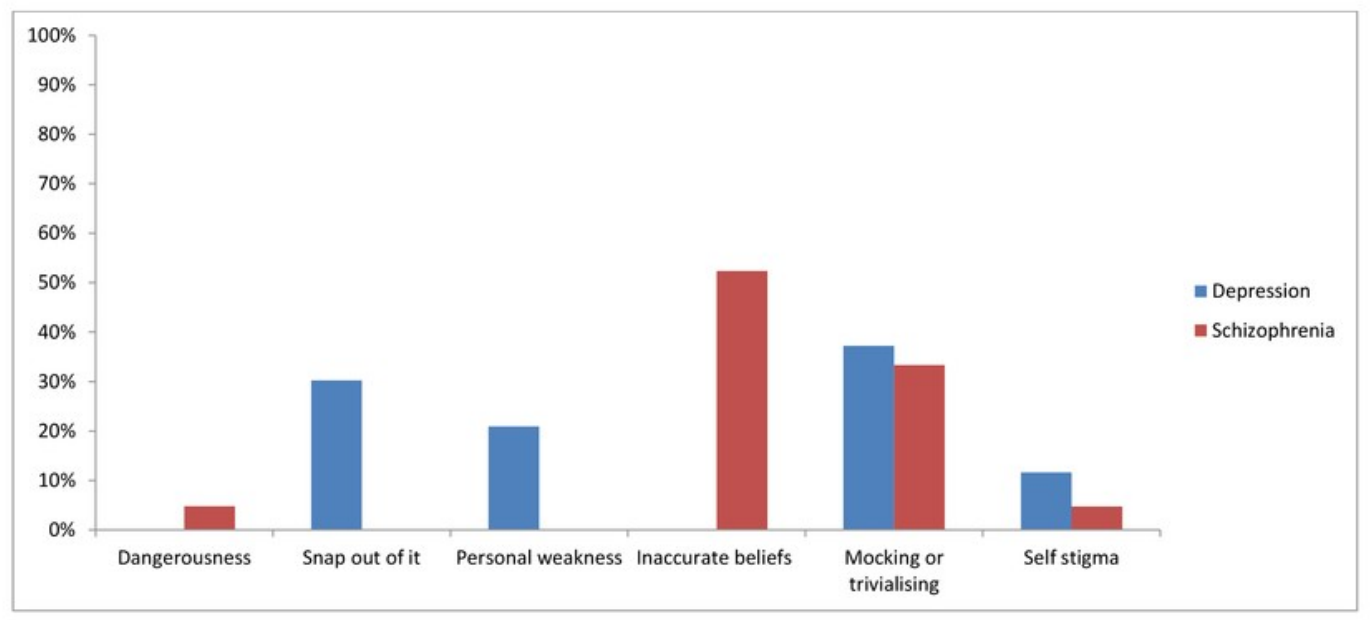

